\title{
FGFR Family Alteration Positive
}

National Cancer Institute

\section{Source}

National Cancer Institute. FGFR Family Alteration Positive. NCI Thesaurus. Code C133705.

Indicates that mutation, overexpression or rearrang ement of an FGFR family member has been detected in a sample. 